\title{
CONVEX NULL SEQUENCE TECHNIQUE FOR ANALYTIC AND UNIVALENT MAPPINGS OF THE UNIT DISK
}

\author{
K. O. BABALOLA
}

\begin{abstract}
In this paper we employ a technique based on the convex null properties of certain infinite sequences to study various classes of analytic and univalent functions in the open unit disk. The technique simplifies many problems of the theory of geometric functions and our results generalize and extend many earlier ones.
\end{abstract}

\section{Introduction}

Let $\Omega$ be the class of functions $\vartheta(z)$ which are regular in the unit disk $E=\{z \in$ $\mathbb{C}:|z|<1\}$ and satisfy $\vartheta(0)=0,|\vartheta(z)|<1$ for $z \in E$. For arbitrary fixed numbers $a, b$ such that $a \in(-1,1]$ and $b \in[-1, a)$, let $P[a, b]$ be the family of functions, $p(z)$, normalized by $p(0)=1$, regular in $E$ and such that for some $\vartheta \in \Omega, p(z)=(1+$ $a \vartheta(z)) /(1+b \vartheta(z)), z \in E$. Equivalently $P[a, b]$ is the family of functions $p(z)$ which are subordinate to $L_{0}(a, b: z)=(1+a z) /(1+b z)$ in the open unit disk $E$.

$P[a, b]$ generalizes the well known family of Caratheodory functions and other classes of functions defined in $E$. For example (see [5]): (i) $P[1,-1] \equiv P$, the class of Caratheodory functions for which $\operatorname{Re} p(z)>0$, (ii) $P[1-2 \beta,-1] \equiv P_{\beta}$, the class of Caratheodory functions of order $\beta, 0 \leq \beta<1$, that is $\operatorname{Re} p(z)>\beta$, (iii) $P[1,1 / \beta-1] \equiv P(\beta)$, the class of functions $p(z)$ satisfying $|p(z)-\beta|<\beta, \beta>1 / 2$, (iv) $P[\beta,-\beta] \equiv P^{(\beta)}$, the class of functions $p(z)$ satisfying $|p(z)-1|<\beta|p(z)+1|, 0<\beta \leq 1$ and (v) $P[\beta, 0] \equiv P_{(\beta)}$, the class of functions $p(z)$ satisfying $|p(z)-1|<\beta, 0<\beta \leq 1$. Furthermore it is also known that $P[a, b] \subseteq P((1-a) /(1-b))$ and $P[a, b] \subseteq P(1 /(1+b))[5]$.

Let $A$ be the class of normalized analytic functions $f(z)=z+a_{2} z^{2}+\cdots$ in $E$. In this article we explore a technique based on the convex null property of certain infinite sequences in the study of some analytic and univalent functions in $E$ : an infinite sequence $c_{0}, c_{1}, \ldots, c_{k}, \ldots$ of nonnegative numbers is said to be a convex null sequence if $c_{k} \rightarrow 0$ as $k \rightarrow \infty$ and $c_{0}-c_{1} \geq c_{1}-c_{2} \geq \cdots \geq c_{k}-c_{k+1} \geq \cdots \geq 0$. To demonstrate our idea, we define new classes of functions using the derivative operators $D^{n}$ and $L_{n}^{\sigma}$ defined, on $A$, in $[2,8]$ as $D^{n} f(z)=z\left[D^{(n-1)} f(z)\right]^{\prime}$ with $D^{0} f(z)=f(z)$, and $L_{n}^{\sigma} f(z)=$ 
$\left(\tau_{\sigma} * \tau_{\sigma, n}^{(-1)} * f\right)(z)$ where $*$ denotes convolution and for any fixed real number $\sigma$ and $n \in \mathbb{N}, \tau_{\sigma, n}(z)=z /(1-z)^{\sigma-(n-1)}, \sigma-(n-1)>0, \tau_{\sigma}=\tau_{\sigma, 0}$ and $\tau_{\sigma, n}^{(-1)}$ is such that $\left(\tau_{\sigma, n} * \tau_{\sigma, n}^{(-1)}\right)(z)=z /(1-z) . D^{n}$ is known as the Salagean derivative operator while $L_{n}^{\sigma}$ was introduced in [2] and includes the well know Ruscheweyh derivative (when $n=\sigma$ ). Now we say:

Definition. Let $\alpha>0$ be a real number and, $n$ and $\sigma$ have their definitions in the operators above. Then a function $f \in A$ is said to belong to the classes $T_{n}^{\alpha}[a, b]$ and $B_{n}^{\sigma}[a, b]$ respectively if and only if

$$
\text { (i) } \frac{D^{n} f(z)^{\alpha}}{\alpha^{n} z^{\alpha}} \in P[a, b], \quad \text { (ii) } \frac{L_{n}^{\sigma} f(z)}{z} \in P[a, b] .
$$

For suitable choices of $a, b$ and $n$, quite a number of subclasses of functions can be deduced from the above definitions.

Associated with the derivative operators are the integrals (respectively): $I_{n} f(z)=$ $I\left(I_{n-1} f(z)\right)=\int_{0}^{z} t^{-1} I_{n-1} f(t) d t$ and $l_{n}^{\sigma} f(z)=\left(\tau_{\sigma}^{(-1)} * \tau_{\sigma, n} * f\right)(z)$ such that $D^{n}\left(I_{n} f(z)\right)=$ $I_{n}\left(D^{n} f(z)\right)=f(z)$ and $L_{n}^{\sigma}\left(l_{n}^{\sigma} f(z)\right)=l_{n}^{\sigma}\left(L_{n}^{\sigma} f(z)\right)=f(z)$. Thus for any $p \in P[a, b]$, nontrivial members of the above classes of functions have the representations: $f(z)=$ $\left\{I_{n}\left[\alpha^{n} z^{\alpha} p(z)\right]\right\}^{1 / \alpha}$ and $f(z)=l_{n}^{\sigma}[z p(z)]$.

In the present study, convex null sequence technique is used to investigate the new classes of functions. First, the technique is applied on some integral transformations of the class $P[a, b]$ and this is presented in the next section. Then, our main results follow very easily and are presented in Sections 3 and 4.

\section{Some integral transformations of $P[a, b]$}

In earlier works $[1,2]$ we have defined the following integral transformations of functions in $P$ as follows (with changes in notations necessary only to unify our discussions): Let $p \in P$. Two nth integral transforms of $p(z), z \in E$ are given as

$$
\phi_{n}^{j}(p(z))=\int_{0}^{z} \lambda_{n}^{j}(z, t) \phi_{n-1}^{j}(p(t)) d t, \quad j=1,2, \quad n \geq 1
$$

where

$$
\lambda_{n}^{1}(z, t)=\alpha \frac{t^{\alpha-1}}{z^{\alpha}}, \quad \alpha>0
$$

and

$$
\lambda_{n}^{2}(z, t)=(\sigma-(n-1)) \frac{t^{\sigma-n}}{z^{\sigma-(n-1)}}, \quad \sigma-(n-1)>0
$$

with $\phi_{0}^{j}(p(z))=p(z)$.

The transformations are iterative and closely associated with certain families analytic and univalent functions involving the well known Salagean and Rucheweyh derivatives 
$[1,2]$. These transformations preserve many geometric structures of the family $P$; particularly the positivity of the real parts, compactness, convexity and subordination. They have been very helpful in dealing easily with certain problems of the theory of analytic and univalent functions.

In the sequel we would apply the above transformations on the family of Janowski functions. The method is simple: replace $P$ in the above definitions by $P[a, b]$ so that for $p \in P[a, b]$, then (1.1) are the desired integrals. We denote by $\Phi_{n}^{j}[a, b]$ the family of transformations of $\phi_{n}^{j}(p(z)), p \in P[a, b]$. If $p(z)=1+c_{1} z+\cdots$, then $\phi_{n}^{j}(p(z))=$ $1+\sum_{k=1}^{\infty} c_{k}^{j} z^{k}$ where $c_{k}^{j}=c_{n, k}^{j} c_{k}$ and

$$
\begin{gathered}
c_{n, k}^{1}=\left(\frac{\alpha}{\alpha+k}\right)^{n}, \\
c_{n, k}^{2}=\frac{\sigma(\sigma-1) \cdots(\sigma-(n-1))}{(\sigma+k)(\sigma+k-1) \cdots(\sigma+k-(n-1))}=\frac{\sigma !}{(\sigma+k) !} \frac{(\sigma+k-n) !}{(\sigma-n) !} .
\end{gathered}
$$

(see $[1,2])$ and it is easy to see that the transformations are analytic, normalized by $\left.\phi_{n}^{j}(p(z))\right|_{z=0}=1$ and $\phi_{n}^{j}(p(z)) \neq 0$ for $z \in E$.

Throughout the paper we would adopt the notations $\phi^{j}$ and $\Phi^{j}, j=1,2$ where we do not require to specify the parameters $n$. Next we present some lemmas which will be relevant in the sequel.

Lemma 1.([3]) Let $\left\{c_{k}\right\}_{k=0}^{\infty}$ be a convex null sequence. Then the function $p(z)=$ $c_{0} / 2+c_{1} z+c_{2} z^{2}+\cdots, z \in E$, is analytic in $E$ and $\operatorname{Re} p(z)>0$.

The next lemma can be derived from the Herglotz representation for $P$ (see also [4]).

Lemma 2. If $p(z)$ is analytic in $E, p(0)=1$ and Re $p(z)>1 / 2, z \in E$, then for any function $q(z)$ analytic in $E$, the convolution $p * q$ takes its values in the convex hull $\overline{\text { of } q}(E)$.

Remark 1. If $\left\{c_{k}\right\}_{k=0}^{\infty}$ is a convex null sequence with $c_{0}=1$, then by Lemma 1, we have $\operatorname{Re} p(z)>1 / 2$. Hence the result of applying such sequence to an arbitrary analytic function is a function which maps the unit disk onto the convex hull of the original image. This is due to Lemma 2.

Remark 2. Since the convex hull of a set is the smallest convex set containing it, then it follows that if $q(z)$ is a convex map, then the convex hull of $q(E)$ is $q(E)$. Hence by Lemmas 1 and 2 a convex null sequence $\left\{c_{k}\right\}_{k=0}^{\infty}$, with $c_{0}=1$, is a subordinating factor sequence for $q(z)$ (see [9]).

Lemma 3. Let $x>1$ be real. If $\zeta \geq 0$, then the following inequality holds

$$
\left(\frac{x}{x-1}\right)^{\zeta}+\left(\frac{x}{x+1}\right)^{\zeta} \geq 2
$$


Proof. Denote the left hand side of the inequality by $h(\zeta)$. Differentiating with respect to $\zeta$, we have

$$
h^{\prime}(\zeta)=\left(\frac{x}{x-1}\right)^{\zeta} \log \left(\frac{x}{x-1}\right)+\left(\frac{x}{x+1}\right)^{\zeta} \log \left(\frac{x}{x+1}\right)
$$

Then we have

$$
\begin{aligned}
h^{\prime}(\zeta) & >\left(\frac{x}{x+1}\right)^{\zeta}\left[\log \left(\frac{x}{x-1}\right)+\log \left(\frac{x}{x+1}\right)\right] \\
& =\left(\frac{x}{x+1}\right)^{\zeta} \log \left(\frac{x^{2}}{x^{2}-1}\right) \\
& >0
\end{aligned}
$$

Hence $h(\zeta)$ is an increasing function of $\zeta$. Thus $h(\zeta) \geq h(0)=2$ for $\zeta \geq 0$. This proves the inequality.

Next we prove:

Theorem 1. $\Phi^{j} \subset P[a, b]$. In other words every transformation $\phi^{j}$ of a $p \in P[a, b]$ is also in $P[a, b]$, that is $\phi^{j}(p(z)) \prec L_{0}(a, b: z)$.

Proof. Let $\phi^{j} \in \Phi^{j}$. Then for any $p \in P[a, b]$ we have to prove that $\phi^{j}(p(z)) \prec$ $L_{0}(a, b: z)$. But $\phi^{j}(p(z))=1+\sum_{k=1}^{\infty} c_{k}^{j} z^{k}$, where $c_{k}^{j}=c_{n, k}^{j} c_{k}$. That is $\phi^{j}(p(z))=$ $(q * p)(z)$ with $q(z)=1+\sum_{k=1}^{\infty} c_{n, k}^{j} z^{k}$. Thus by Lemma $2, \phi^{j}$ maps the open unit disk onto the convex hull of the original image, $p(E)$, if $\operatorname{Re} q(z)>1 / 2$. This follows from Lemma 1 if we prove that the sequence $\left\{c_{n, k}^{j}\right\}_{k=0}^{\infty}$ is convex null for each $j=1,2$. It is obvious that for $k=0,1, \cdots, c_{n, k}^{j}>0$ and $c_{n, k}^{j} \rightarrow 0$ as $k \rightarrow \infty$. We need to prove that $c_{n, k}^{j}-2 c_{n, k+1}^{j}+c_{n, k+2}^{j} \geq 0, k=0,1, \cdots$. Equivalently, we will prove that $\Lambda_{j}=c_{n, k}^{j} / c_{n, k+1}^{j}+c_{n, k+2}^{j} / c_{n, k+1}^{j} \geq 2$. By simple calculations we have

$$
\begin{aligned}
& \Lambda_{1}=\left(\frac{\alpha+k+1}{\alpha+k}\right)^{n}+\left(\frac{\alpha+k+1}{\alpha+k+2}\right)^{n} \geq 2 . \\
& \Lambda_{2}=2+\frac{n(n+1)}{(\sigma+k+1-n)(\sigma+k+2)} \geq 2 .
\end{aligned}
$$

That $\Lambda_{1} \geq 2$ follows from Lemma 3 by taking $x=\alpha+k+1$. Thus the proof is complete.

Theorem 2. $\Phi_{n+1}^{j}[a, b] \subset \Phi_{n}^{j}[a, b], n \in \mathbb{N}$.

Proof. Let $p \in P[a, b]$. We have to prove that if $\phi_{n}^{j}(p(z)) \prec L_{0}(a, b: z)$, then $\phi_{n+1}^{j}(p(z)) \prec L_{0}(a, b: z)$. Suppose $\phi_{n+1}^{j} \in \Phi_{n+1}^{j}[a, b]$. Then by simple calculations we find that the coefficients $c_{n+1, k}^{j}$ of $\phi_{n+1}^{j}(p(z))$ can be decomposed as $c_{n+1, k}^{1}=c_{1, k}^{1} c_{n, k}^{1}$ 
and $c_{n+1, k}^{2}=((\sigma-n) /(\sigma+k-n)) c_{n, k}^{2}$ so that

$$
\begin{aligned}
\phi_{n+1}^{1}(p(z)) & =1+\sum_{k=1}^{\infty} c_{n+1, k}^{1} z^{k} \\
& =\left(1+\sum_{k=1}^{\infty} c_{1, k}^{1} z^{k}\right) *\left(1+\sum_{k=1}^{\infty} c_{n, k}^{1} z^{k}\right)
\end{aligned}
$$

and

$$
\begin{aligned}
\phi_{n+1}^{2}(p(z)) & =1+\sum_{k=1}^{\infty} c_{n+1, k}^{2} z^{k} \\
& =\left(1+\sum_{k=1}^{\infty} \frac{\sigma-n}{\sigma-n+k} z^{k}\right) *\left(1+\sum_{k=1}^{\infty} c_{n, k}^{2} z^{k}\right) .
\end{aligned}
$$

Hence relying on the convex-nullity of sequences $\left\{c_{1, k}^{1}\right\}_{k=0}^{\infty}$ and $\left\{\frac{\sigma-n}{\sigma-n+k}\right\}_{k=0}^{\infty}$, which can be easily verified as in Theorem 1 , we conclude that $\phi_{n+1}^{j}(p(z)), j=1,2$, maps $E$ onto the convex hull of the image of $E$ under $\phi_{n}^{j}(p(z))$. That is for all $n \in \mathbb{N}, j=1,2$, $\phi_{n}^{j}(p(z)) \prec L_{0}(a, b: z)$ implies $\phi_{n+1}^{j}(p(z)) \prec L_{0}(a, b: z)$ as required and the proof is complete.

Theorem 3. Let $\phi^{j} \in \Phi^{j}$. Define

$$
M_{n}^{j}(a, b ; r)=1+(a-b) \sum_{k=1}^{\infty}(-b)^{k-1} c_{n, k}^{j} r^{k}, \quad|z|=r
$$

and

$$
m_{n}^{j}(a, b ; r)=1+(a-b) \sum_{k=1}^{\infty} b^{k-1} c_{n, k}^{j}(-r)^{k}, \quad|z|=r .
$$

Then $m_{n}^{j}(a, b ; r) \leq R e \phi^{j} \leq\left|\phi^{j}\right| \leq M_{n}^{j}(a, b ; r)$. Lower bound equality is attained by $\phi^{j}\left(L_{0}(a, b:-z)\right)$ while equality in the upper bound is attained by $\phi^{j}\left(L_{0}(a, b: z)\right)$.

Proof. Let $p \in P[a, b]$ and define $z=r e^{i \theta}$ and $t=\rho e^{i \theta}, 0<\rho \leq r<1$. The rest of the proof is similar to that presented in [1] if we use the known inequalities, Re $p\left(r e^{i \theta}\right) \geq(1-a r) /(1-b r)$ and $\left|p\left(r e^{i \theta}\right)\right| \leq(1+a r) /(1+b r)$.

Corollary 1. $\phi^{j} \in \Phi^{j}$ if and only if $\phi^{j}(p(z)) \prec \phi^{j}\left(L_{0}(a, b: z)\right)$ where

$$
\phi_{n}^{j}\left(L_{0}(a, b: z)\right)=\int_{0}^{z} \lambda_{n}^{j}(z, t) \phi_{n-1}^{j}\left(L_{0}(a, b: t)\right) d t, \quad j=1,2, \quad n \geq 1 .
$$

$\phi^{j}\left(L_{0}(a, b: z)\right)$ is the best dominant.

Proof. Suppose $\phi^{j} \in \Phi^{j}$. Then by Theorem $3, \phi^{j}(p(E)) \subseteq \phi^{j}\left(L_{0}(a, b: E)\right)$ and furthermore $\left.\phi^{j}(p(z))\right|_{z=0}=\left.\phi^{j}\left(L_{0}(a, b: z)\right)\right|_{z=0}$, which is a well known subordination condition. Thus we have $\phi^{j}(p(z)) \prec \phi^{j}\left(L_{0}(a, b: z)\right)$. 
On the other hand, suppose that $\phi^{j}(p(z)) \prec \phi^{j}\left(L_{0}(a, b: z)\right)$. Then by the convex null property of the coefficients $c_{n, k}^{j}, \phi^{j}\left(L_{0}(a, b: z)\right)$ maps $E$ onto the convex hull of $L_{0}(a, b: E)$ (which is $L_{0}(a, b: E)$ since $L_{0}(a, b: z)$ is convex univalent in $E$ by Remark $2)$. Thus $\phi^{j}\left(L_{0}(a, b: z)\right) \prec L_{0}(a, b: z)$ and the conclusion follows. That $\phi^{j}\left(L_{0}(a, b: z)\right)$ is the best dominant is a consequence of the fact that equality in Theorem 3 is attained by it.

The above corollary leads to:

Remark 3. The following statements are equivalent:

(i) $p(z) \prec L_{0}(a, b: z)$

(ii) $p \in P[a, b]$

(iii) $\phi^{j} \in \Phi^{j}[a, b]$

(iv) $\phi^{j}(p(z)) \prec \phi^{j}\left(L_{0}(a, b: z)\right)$

\section{Properties of $\boldsymbol{T}_{n}^{\alpha}[\boldsymbol{a}, \boldsymbol{b}]$}

Let all parameters having the usual definitions. Also let $p \in P[a, b], p(z)=1+c_{1} z+$ $\cdots$, then from definitions and the integral representations, we have the following lemma:

Lemma 4. Let $f \in A$. Then the following are equivalent: (i) $f \in T_{n}^{\alpha}[a, b]$, (ii) $\left(D^{n} f(z)^{\alpha}\right) /\left(\alpha^{n} z^{\alpha}\right) \in P[a, b]$ and (iii) $f(z)^{\alpha} / z^{\alpha} \in \Phi_{n}^{1}[a, b]$.

Theorem 4. If $f \in T_{n}^{\alpha}[a, b]$, then $f(z)^{\alpha} / z^{\alpha} \prec(1+a z) /(1+b z)$.

Proof. Let $f \in T_{n}^{\alpha}[a, b]$. Then by Lemma $4, f(z)^{\alpha} / z^{\alpha} \in \Phi_{n}^{1}[a, b]$. Thus by Theorem 1 , we have $f(z)^{\alpha} / z^{\alpha} \in P[a, b]$.

Theorem 5. For $n \in \mathbb{N}, T_{n+1}^{\alpha}[a, b] \subset T_{n}^{\alpha}[a, b]$.

Proof. Let $f \in T_{n+1}^{\alpha}[a, b]$. Then by Lemma $4, f(z)^{\alpha} / z^{\alpha} \in \Phi_{n+1}^{1}[a, b]$. By Theorem $2, f(z)^{\alpha} / z^{\alpha} \in \Phi_{n}^{1}[a, b]$. By Lemma 4 again $f \in T_{n}^{\alpha}[a, b]$.

Corollary 2. For all $n \geq 1, T_{n}^{\alpha}[a, b]$ consist only of univalent functions in $E$.

Proof. For $n \geq 1$, by Theorem 5 we have $T_{n}^{\alpha}[a, b] \subset T_{1}^{\alpha}[a, b]$. However $T_{1}^{\alpha}[a, b]$ consists of functions $f \in A$ such that $f(z)^{\alpha-1} f^{\prime}(z) / z^{\alpha-1} \in P[a, b] \subseteq P((1-a) /(1-b))$. This implies that $\operatorname{Re} f(z)^{\alpha-1} f^{\prime}(z) / z^{\alpha-1}>0$, which is sufficient for univalence in $E$ (see [1]).

Using Lemma 4, we have the following growth properties of functions in $T_{n}^{\sigma}[a, b]$ by choosing $\phi^{1}=f(z)^{\alpha} / z^{\alpha}$ in Theorem 3 .

Theorem 6. Let $f \in T_{n}^{\alpha}[a, b]$. Then

$$
m_{n}^{1}(a, b ; r) \leq R e \frac{f(z)^{\alpha}}{z^{\alpha}} \leq\left|\frac{f(z)^{\alpha}}{z^{\alpha}}\right| \leq M_{n}^{1}(a, b ; r) .
$$


Lower bound equality is attained by $f(z)=\left\{I_{n}\left[\alpha^{n} z^{\alpha} L_{0}(a, b:-z)\right]\right\}^{1 / \alpha}$ while upper bound equality is realised by $f(z)=\left\{I_{n}\left[\alpha^{n} z^{\alpha} L_{0}(a, b: z)\right]\right\}^{1 / \alpha}$.

Theorem 7. $f \in T_{n}^{\alpha}[a, b] \Leftrightarrow f(z)^{\alpha} / z^{\alpha} \prec 1+(a-b) \sum_{k=1}^{\infty}(-b)^{k-1} c_{n, k}^{1} z^{k}$. The function on the right hand side of subordination is the best dominant.

Proof. By definition, $f \in T_{n}^{\alpha}[a, b] \Leftrightarrow\left(D^{n} f(z)^{\alpha}\right) /\left(\alpha^{n} z^{\alpha}\right) \prec(1+a z) /(1+b z) \Leftrightarrow$ there exists a function $p(z)=1+c_{1} z+\cdots$ in $P[a, b]$ such that $\left(D^{n} f(z)^{\alpha}\right) /\left(\alpha^{n} z^{\alpha}\right)=1+c_{1} z+\cdots$ $\Leftrightarrow f(z)^{\alpha} / z^{\alpha}=1+\sum_{k=1}^{\infty}\left(\frac{\alpha}{\alpha+k}\right)^{n} c_{k} z^{k}$. If we set $\phi^{1}(z)=f(z)^{\alpha} / z^{\alpha}$ in Corollary 1 we have the subordination.

Next we consider two integral transforms within the classes $T_{n}^{\alpha}[a, b]$. Let $\alpha>0$, and $\gamma \geq 0$ be real numbers. Define $\mathcal{J}_{0}^{j}(z)=f(z), j=1,2$ and for $\zeta>0$ define

and

$$
\mathcal{J}_{\zeta}^{1}(f)=\left\{\frac{(\alpha+\gamma)^{\zeta}}{z^{\gamma} \Gamma(\zeta)} \int_{0}^{z}\left(\log \frac{z}{t}\right)^{\zeta-1} t^{\gamma-1} f(t)^{\alpha} d t\right\}^{\frac{1}{\alpha}}
$$

$$
\mathcal{J}_{\zeta}^{2}(f)=\left\{\left(\begin{array}{c}
\alpha+\gamma+\zeta-1 \\
\alpha+\gamma-1
\end{array}\right) \frac{\zeta}{z^{\gamma}} \int_{0}^{z}\left(1-\frac{t}{z}\right)^{\zeta-1} t^{\gamma-1} f(t)^{\alpha} d t\right\}^{\frac{1}{\alpha}}
$$

where $\zeta-1+\gamma \geq 0$.

Theorem 8. The classes $T_{n}^{\alpha}[a, b]$ are closed under $\mathcal{J}_{\zeta}^{j}(f)$.

Proof. Let $f(z)=z+a_{2} z^{2}+\cdots \in T_{n}^{\alpha}[a, b]$. For $\alpha>0$, we can write

$$
f(z)^{\alpha}=z^{\alpha}+A_{2}(\alpha) z^{\alpha+1}+\cdots
$$

where $A_{k}(\alpha), k=2,3, \ldots$, depends on the coefficients $a_{k}$ of $f(z)$ and the index $\alpha$. Thus evaluating the integrals in series form, also using the Beta and Gamma functions and noting that

$$
\left(\begin{array}{c}
\zeta \\
\gamma
\end{array}\right)=\frac{\Gamma(\zeta+1)}{\Gamma(\zeta-\gamma+1) \Gamma(\gamma+1)}
$$

we obtain $\mathcal{J}_{\zeta}^{j}(f)^{\alpha}=z^{\alpha}+\sum_{k=1}^{\infty} C_{k}^{j} A_{k+1}(\alpha) z^{\alpha+k}$ where

$$
C_{k}^{1}=\left(\frac{\alpha+\gamma}{\alpha+\gamma+k}\right)^{\zeta} \text { and } C_{k}^{2}=\frac{\Gamma(\alpha+\gamma+\zeta)}{\Gamma(\alpha+\gamma)} \frac{\Gamma(\alpha+\gamma+k-1)}{\Gamma(\alpha+\gamma+\zeta+k)}
$$

Now we have

$$
\begin{aligned}
\frac{\mathcal{J}_{\zeta}^{j}(f)^{\alpha}}{z^{\alpha}} & =1+\sum_{k=1}^{\infty} C_{k}^{j} A_{k+1}(\alpha) z^{k} \\
& =\left(1+\sum_{k=1}^{\infty} C_{k}^{j} z^{k}\right) *\left(1+\sum_{k=1}^{\infty} A_{k+1}(\alpha) z^{k}\right) \\
& =Q(z) * \frac{f(z)^{\alpha}}{z^{\alpha}}
\end{aligned}
$$


where $Q(z)=1+\sum_{k=1}^{\infty} C_{k}^{j} z^{k}$. By calculations, we find that

$$
\begin{gathered}
\frac{C_{k}^{1}}{C_{k+1}^{1}}+\frac{C_{k+2}^{1}}{C_{k+1}^{1}}=\left(\frac{\alpha+\gamma+k+1}{\alpha+\gamma+k}\right)^{\zeta}+\left(\frac{\alpha+\gamma+k+1}{\alpha+\gamma+k+2}\right)^{\zeta} \geq 2 . \\
\frac{C_{k}^{2}}{C_{k+1}^{2}}+\frac{C_{k+2}^{2}}{C_{k+1}^{2}}=2+\frac{\zeta(\zeta+1)}{(\alpha+\gamma+k+1)(\alpha+\gamma+\zeta+k+2)} \geq 2 .
\end{gathered}
$$

Note that the first inequality is a consequence of Lemma 3, where $x=\alpha+\gamma+k+1$. Furthermore for each $k=0,1, \cdots, C_{k}^{j}>0$ and $C_{k}^{j} \rightarrow 0$ as $k \rightarrow \infty$, hence the sequences $\left\{C_{k}^{j}\right\}_{0}^{\infty}, j=1,2$ are convex null. Thus $\mathcal{J}_{\zeta}^{1}(f)^{\alpha} / z^{\alpha}$ maps the open unit disk $E$ onto the convex hull of $f(z)^{\alpha} / z^{\alpha}$ and by Theorem 7 our conclusion follows.

\section{Properties of $\boldsymbol{B}_{n}^{\boldsymbol{\sigma}}[\boldsymbol{a}, \boldsymbol{b}]$}

In this section, we state without proofs, the analogue of the results of the preceeding section for the classes $B_{n}^{\sigma}[a, b]$. The results are consequences of the following lemma and an appropriate choice $\phi^{2}=f(z) / z$.

Lemma 5. Let $f \in A$. Then the following are equivalent: (i) $f \in B_{n}^{\sigma}[a, b]$, (ii) $\left(L_{n}^{\sigma} f(z)\right) / z \in P[a, b]$ and (iii) $f(z) / z \in \Phi_{n}^{2}[a, b]$.

Theorem 9. If $f \in B_{n}^{\sigma}[a, b]$, then $f(z) / z \prec(1+a z) /(1+b z)$.

Theorem 10. For $n \in \mathbb{N}, B_{n+1}^{\sigma}[a, b] \subset B_{n}^{\sigma}[a, b]$.

Corollary 3. For all $n \geq 1, B_{n}^{\sigma}[a, b]$ consist only of univalent functions in $E$.

Theorem 11. Let $f \in B_{n}^{\sigma}[a, b]$. Then

$$
m_{n}^{2}(a, b ; r) \leq R e \frac{f(z)}{z} \leq\left|\frac{f(z)}{z}\right| \leq M_{n}^{2}(a, b ; r) .
$$

Lower bound equality is attained by $f(z)=l_{n}^{\sigma}\left[z L_{0}(a, b:-z)\right]$ while upper bound equality is realised by $f(z)=l_{n}^{\sigma}\left[z L_{0}(a, b: z)\right]$.

Theorem 12. $f \in B_{n}^{\sigma}[a, b] \Leftrightarrow f(z) / z \prec 1+(a-b) \sum_{k=1}^{\infty}(-b)^{k-1} c_{n, k}^{2} z^{k}$. The analytic function on the right hand side is the best dominant.

Theorem 13. The classes $B_{n}^{\sigma}[a, b]$ are closed under $\mathcal{J}_{\zeta}^{j}(f), \alpha=1$.

\section{Acknowledgements}

This work was carried out at the Centre for Advanced Studies in Mathematics, CASM, Lahore University of Management Sciences, Lahore, Pakistan during the author's postdoctoral fellowship at the Centre. The author is indebted to all staff of CASM for their hospitality, most especially Prof. Ismat Beg. 


\section{References}

[1] Babalola, K. O. and Opoola, T. O., Iterated integral transforms of Caratheodory functions and their applications to analytic and univalent functions, Tamkang J. Math. 37 (Winter 2006), 355-366.

[2] Babalola, K. O., New subclasses of analytic and univalent function involving certain convolution operators, Mathematica (Cluj), Tome 50 (73), No. 1 (2008), 3-12.

[3] Fejer, L., Uber die positivat von summen, die nach trigonometrischen oder Legendreschen funktion fortschreiten I, Acta Szeged 2 (1925), 75-86(German).

[4] Goodman, A. W., Univalent Functions, Vols. I \& II, Marina Pub. Co., Tampa, Florida, 1993.

[5] Janowski, W., Some extremal problems for certain families of analytic functions I, Ann. Polon. Math. 28 (1973), 297-326.

[6] Jung, I. B., Kim, Y. C. and Srivastava, H. M., The Hardy space of analytic functions associated with certain one-parameter families of integral operators, J. Math. Anal. Appl. 176, (1993), 138-147.

[7] Macgregor, T. H., Functions whose derivatives have positive real part, Trans. Amer. Math. Soc. 104 (1962), 532-537.

[8] Salagean, G. S., Subclasses of univalent functions, Lecture Notes in Math. 1013 (1983), 362-372. Springer-Verlag, Berlin, Heidelberg and New York.

[9] Wilf, H. S., Subordinating factor sequences for convex maps of the unit circle, Proc. Amer. Math. Soc. 12 (1961), 689-693.

Current Address: Centre for Advanced Studies in Mathematics, Lahore University of Management Sciences, Lahore, Pakistan.

E-mail: kobabalola@lums.edu.pk

Permanent Address: Department of Mathematics, University of Ilorin, Ilorin, Nigeria.

E-mail: khayrah.babalola@gmail.com 